\title{
Liver injury changed the biological characters of serum small extracellular vesicles and reprogramed hepatic macrophages in mice
}

\section{Xiufang Lv}

Department of Pathology, Medical School of Nantong University, Nantong, 226001, China; Key Laboratory of Microenvironment and Translational Cancer Research, Nantong, 226001, China https://orcid.org/0000-0003-2345-848X

\section{Jing Li}

Department of Pathology, Medical School of Nantong University, Nantong, 226001, China. Department of Pathology, National Cancer Center/National Clinical Research Center for Cancer/Cancer Hospital, Chinese Academy of Medical Sciences and Peking Union Medica

\section{Li He}

Department of Pathology, Medical School of Nantong University, Nantong, 226001, China. Department of Pathology, Nanjing Jinling Hospital, Nanjing University School of Medicine, Nanjing, 210093, China Li Cheng

Department of Pathology, Medical School of Nantong University, Nantong, 226001, China. Department of Pathology, Affiliated Jiangyin Hospital of Southeast University Medical College, Jiangyin, 214400, China

\section{Min Zhao}

Department of Pathology, Medical School of Nantong University, Nantong, 226001, China. Key Laboratory of Microenvironment and Translational Cancer Research, Nantong, 226001, China

\section{Weiqi Liu}

Department of Pathology, Medical School of Nantong University, Nantong, 226001, China. Key Laboratory of Microenvironment and Translational Cancer Research, Nantong, 226001, China

\section{Anqi Zhang}

Department of Pathology, Medical School of Nantong University, Nantong, 226001, China. Key Laboratory of Microenvironment and Translational Cancer Research, Nantong, 226001, China Yufeng Sun

Department of Pathology, Medical School of Nantong University, Nantong, 226001, China. Key Laboratory of Microenvironment and Translational Cancer Research, Nantong, 226001, China

\section{Gang Qin}

Center for Liver Diseases, the Third Affiliated Hospital of Nantong University, Nantong, 226006, China Peng Lu 
Department of Pathology, Medical School of Nantong University, Nantong, 226001, China. Key Laboratory of Microenvironment and Translational Cancer Research, Nantong, 226001, China

\section{Yuhua Ji}

Key Laboratory of Neuroregeneration of Jiangsu and Ministry of Education, Nantong University, Nantong, 226001, China. Institute of Immunology, College of Life Science and Technology, Jinan University, Guangdong, 510632, China

\section{Juling Ji ( $\square$ jijuling@ntu.edu.cn )}

Department of Pathology, Medical School of Nantong University, Nantong, 226001, China. Key Laboratory of Microenvironment and Translational Cancer Research, Nantong, 226001, China https://orcid.org/0000-0001-6500-8052

\section{Research}

Keywords: microRNA, small RNA sequencing, biomarker, monocyte-derived macrophage, resident macrophage

Posted Date: January 13th, 2021

DOI: https://doi.org/10.21203/rs.3.rs-142242/v1

License: (c) (1) This work is licensed under a Creative Commons Attribution 4.0 International License. Read Full License 


\section{Abstract \\ Background}

Serum small extracellular vesicles (sEVs) and their small RNA (sRNA) cargoes could be promising biomarkers for the diagnosis of liver injury. However, the dynamic changes of serum sEVs and their sRNA components during liver injury and the biological functions of these liver-injury-serum sEVs have not been well characterized.

\section{Methods}

To identify serum sEV biomarkers for liver injury, we established a carbon tetrachloride-induced mouse liver injury model to simulate acute liver injury (ALI), chronic liver injury (CLI) and recovery. Serum sEVs were obtained and characterized. Serum sEV sRNAs were profiled. Differentially expressed microRNAs (miRNA) were compared to mouse liver enriched miRNAs and previously reported circulating miRNAs that related to human liver diseases. The biological significance was evaluated by Ingenuity Pathway Analysis of altered sEV miRNAs, and conditional culture of ALI serum sEVs with primary hepatic macrophages.

\section{Results}

We found that both ALI and CLI changed the concentration and morphology of serum sEVs. The proportion of serum sEV miRNA increased upon liver injury, with the liver as the primary contributor. The altered serum sEV miRNAs based on mice's study were consistent with those human liver diseasesrelated circulating miRNAs. Serum sEV miRNA signatures for ALI and CLI, and a panel of miRNAs as common marker for liver injury, were established. The differential serum sEV miRNAs in ALI mainly contributed to liver steatosis and inflammation, while those in CLI primarily contributed to hepatocellular carcinoma and hyperplasia. ALI serum sEVs decreased both CD86 and CD206 expression in monocytederived macrophages, but increased CD206 expression in resident macrophages.

\section{Conclusion}

Serum sEVs in the different stages of liver injury carried different sRNA messages and contributed to diverse pathological processes. ALI serum sEVs might alleviate liver damage by depolarizing monocytederived macrophages and educating resident liver macrophage to M2 like cells.

\section{Background}

Because of its strategic location and bio-functions, the liver is particularly susceptible to various pathogenic factors, including drugs, alcohol, and viruses. The initial insult induces acute liver injury (ALI) or even liver failure. Repeated or persistent insults will cause chronic liver injury (CLI) and result in liver 
fibrosis and finally fatal cirrhosis [1,2]. So it is important to identify individuals with liver injury. But the liver injury does not always cause noticeable signs and symptoms. Except for the wildly used blood liver function tests on the serum alanine aminotransferases (ALT) and aspartate aminotransferase (AST), which are not always restricted to liver injury [3], there is still a need to explore specific and sensitive biomarkers. With the rapid progress in medical research, it is now possible and necessary to search for new biomarkers from serum small extracellular vesicles (sEVs).

Recently, serum sEVs attracted tremendous interest due to their essential roles in intercellular communication and diagnostic and therapeutic potential [4]. The term sEVs refers to extracellular vesicles released by cells with relatively small size $(<200 \mathrm{~nm})$, which are previously regarded as exosomes [5]. The cargoes carried by sEVs represent a snapshot of the parental cells at the time of release and change depending on the physiological and pathological states [6, 7]. In the liver, sEVs are released from both hepatocytes and nonparenchymal cells to the extracellular space and circulation. Several studies reported abnormally expressed circulating sEV RNAs or proteins for drug-induced liver injury, steatohepatitis, viral-hepatitis, and hepatocellular carcinoma (HCC) $[6,8]$.

The microRNAs (miRNAs) are 22-24 nt small non-coding RNAs involved in post-transcriptional regulation and various biological processes [9]. Tissue-specific distribution is a key feature of miRNAs, making them good candidates as biomarkers or therapeutic targets for particular tissue injury [10-12]. Serum miRNAs have been studied in a variety of liver diseases [13]. However, compared to serum miRNAs, serum sEV miRNAs are well-protected from RNA enzymes. Thus, serum sEV can serve as a more reliable miRNA pool [14]. We proposed that serum sEVs and their miRNA cargoes might reflect the damage of the liver upon injury and could be promising biomarkers.

In the present study, we tried to determine the effects of the liver injury on serum sEVs and the small RNAs (sRNAs) they transported; we were also interested to know if there is any difference between acute and chronic injury. A study in this regard will help us to identify potential serum sEV miRNA biomarkers. The dynamic changes in the number and morphology and sRNA components of serum sEVs were examined. The profiles of deregulated serum sEV miRNAs were obtained and compared to mouse liver enriched miRNAs and previously reported circulating miRNAs related to human liver diseases. To further evaluate the biological significance of the serum SEVs upon liver injury, conditioned cultures of ALI serum sEVs and primary hepatic macrophages were carried out.

\section{Materials And Methods}

\section{Animal studies}

Male C57BL/6 mice (8 weeks) were purchased from Shanghai medical laboratory animal center. For acute liver injury (ALI), mice were treated with a single dose of $\mathrm{CCL}_{4}(0.5 \mathrm{~mL} / \mathrm{kg}$ intraperitoneal injection) dissolved in olive oil (1:9). Mice were sacrificed at 2 or 7 days. For chronic liver injury (CLI), mice were treated with $\mathrm{CCL}_{4}(0.5 \mathrm{~mL} / \mathrm{kg})$ or vehicle twice a week for eight weeks [15]. Mice were sacrificed 48 hours 
after the last injection in 8 weeks or at 12 weeks (Fig. 1A). Mice treated with the same volume of olive oil served as the control for ALI or CLI model. Blood or livers were collected from each group for further analyses.

\section{Liver function test and histopathologic examination}

Blood was collected by left ventricular puncture from mice and was left undisturbed for 1 hour at $37^{\circ} \mathrm{C}$, and 2 hours at $4^{\circ} \mathrm{C}$. Afterward, samples were centrifuged at $1000 \mathrm{~g}$ for $10 \mathrm{~min}$ at $4{ }^{\circ} \mathrm{C}$; the clear upper fractions were aliquoted and stored at $-80^{\circ} \mathrm{C}$. Serum ALT and AST levels were measured on an ADVIA 1800 autoanalyzer (Siemens Healthcare Diagnostics, Deerfield, IL, USA). Livers were preserved in 4\% paraformaldehyde, then paraffin-embedded and sectioned. Liver tissue sections were stained with hematoxylin and eosin (Beyotime Biotechnology, Shanghai, China) for routine histology and $0.1 \%$ Sirius Red (Sigma-Aldrich, St. Louis, MO, USA) for collagen.

\section{Mouse serum sEVs isolation and characterization}

Exosomes-enriched serum sEV fractions were precipitated using ultracentrifugation method and ExoQuick precipitation kit (System Biosciences Inc., Mountain View, CA) [16]. The size and particle concentration of the isolated serum sEVs were measured by NTA (NanoSight NS300, Malvern, UK). Serum sEVs were visualized using transmission electron microscopy (TEM, HT7700, Hitachi Ltd., Tokyo, Japan). The expression of exosomal protein markers were determined by Western blots. Details are provided in the Supporting Information.

\section{Small RNA library construction and deep sequencing}

Serum sEV small RNA-seq was conducted by BMK Biotech Co., Ltd. (Beijing, China) with biological replicates for each group.

\section{Sequencing data analysis and bioinformatic analysis}

Raw data were processed as described previously [16]. Trimmed sequencing reads were deposited in the European Nucleotide Archive (https://www.ebi.ac.uk/arrayexpress/experiments/E-MTAB-9462). The bioinformatic analysis of differentially expressed serum sEV miRNAs was performed using Ingenuity Pathways Analysis (QIAGEN, Valencia, CA, USA). The significance of gene enrichment with a particular biologically relevant function was determined by a one-sided Fisher's exact test.

\section{Systemic review for abnormally expressed circulating miRNAs in human liver diseases}

The detailed procedure was provided in the Supporting Information.

\section{Isolation and culture of mouse hepatic macrophages}

Primary mouse hepatic macrophages were isolated from male C57BL/ 6 mice liver by Percoll (GE Healthcare, Princeton, NJ, USA) density gradient centrifugation. Incubation of liver macrophage with mice 
serum sEVs and subsequent multiple-color flow cytometric analysis were carried out. Details are provided in the Supporting Information.

\section{Statistical analysis}

Statistical analyses were performed with GraphPad Prism 7.0 software (GraphPad Software, Inc., La Jolla, CA). Quantitative data were reported as the mean \pm SD. Comparisons between groups were made by Student's t-test or one-way ANOVA. All P values were two-sided and the statistical significance was defined as less than 0.05. Except for small RNA-seq experiments, which included two biological replicates for each group, the data provided in the present study were from three or more independent experiments.

Additional methods and details are provided in the Supporting Information.

\section{Results}

\section{Establishment and validation of $\mathrm{CCL}_{4}$-induced $\mathrm{ALI}$ and $\mathrm{CLI}$ in mice.}

$\mathrm{ALI}$ and recovery model, as well as $\mathrm{CLI}$ and the recovery model of mice induced by $\mathrm{CCL}_{4}$, were established and validated (Fig. 1).

In acutely injured mice (2D), serum ALT and AST levels increased, hepatocyte necrosis and inflammatory cell infiltration can be observed around the lobular central vein. After five days of recovery (7D), the elevated ALT and AST levels returned to the baseline of control for ALI (AC) group, and histological changes were also recovered (Fig. 1A-B). For CLI, repeated $\mathrm{CCL}_{4}$ treatment induced a slight elevation of serum ALT (8W) and then recovered to baseline of control for CLI (CC) 4 weeks after the cease of $\mathrm{CCL}_{4}$ treatment (12W) (Fig. 1C). Damaged hepatocytes and centrilobular contracture can be found in the liver of $\mathrm{CLI}(8 \mathrm{~W})$, with mild inflammatory cell infiltration. Sirius red staining showed obviously collagen deposition and formation of pseudolobules in $8 \mathrm{~W}$ CLI mice. These morphological changes were alleviated in recovery mice (12W) (Fig. 1D).

\section{Identification and characterization of serum sEVs from ALI and CLI mice.}

The isolated particles were spherical or cup-shaped as observed by transmission electron microscopy (TEM) (Fig. 2A); the exosomal protein markers, including CD63, CD81, and CD9, were all highly expressed (Fig. 2B) as determined by Western blots.

Nanoparticles tracking analysis (NTA) showed that the mean diameter of the particles ranged from 90.2 to $127.8 \mathrm{~nm}$. Compared with control group; the number of particles increased in 2D group, but decreased in 7D group; the particle diameters were smaller in 2D group, but larger in 7D group. We also noticed that the size distribution of sEVs widened with multiple peaks in the 7D group (Fig. 2C). For CLI mice, compared with the control group, the particle concentrations of $8 \mathrm{~W}$ and $12 \mathrm{~W}$ groups showed a trend of reduction, although there was no statistical difference. The size distribution of sEVs expanded with multiple peaks in both $8 \mathrm{~W}$ and $12 \mathrm{~W}$ groups, and the particle diameters were larger in both $8 \mathrm{~W}$ and $12 \mathrm{~W}$ 
groups (Fig. 2D). The TEM examination revealed that the multiple peaks reflected the aggregation or fusion of mice serum sEVs presented in 7D, 8W, and 12W samples (Fig. 2C-D).

These findings suggested that both ALI and CLI changed the number and morphology of mice serum sEVs, and even when the visible histological changes of liver recovered in 7D and 12W, the changes in particle number and morphology of serum sEVs persisted.

\section{Comparison of the sRNA components in serum sEVs from ALI and CLI mice.}

A dramatic increase in both total RNA and sRNA contents was observed in serum sEVs upon ALI (2D (Fig. 3A-B). For CLI, there was no significant difference in either sEVs RNA or small RNA contents among each group. RNA sequencing (RNA-seq) and annotation revealed that each pair of biological repeats had at least $94.97 \%$ common sequences in clean reads (Fig S1). In the control groups (AC and CC), tRNA was the dominant sRNA species in serum sEVs, followed by rRNA and miRNA (Fig. 3C). A most remarkable change of serum sEVs sRNAs was the increase of miRNA proportion in both ALI and CLI mice. Compared to the control groups, the proportion of miRNAs increased to more than four folds higher upon ALI, and then returned to baseline in 7D; the proportion of miRNAs increased to almost three folds in CLI mice, and later partially recovered in $12 \mathrm{~W}$ group. With the increase of miRNA, the proportion of tRNA decreased (Fig. 3D).

\section{The miRNA expression profiles of serum sEVs from ALI and CLI mice.}

For ALI and CLI groups, 467 and 488 detectable sEVs (transcript per million reads, TPM $\geq 5.0$ ) miRNAs were obtained, respectively. The biological replications were highly correlated in each group (Fig. 4A). RNA-seq data were further validated by qRT-PCR. Differentially expressed miRNAs of different abundance were selected for validation (Fig. 4B).

Compared to the AC group, 91 miRNAs were up-regulated, and 85 miRNAs were down-regulated (Fold Change $\geq 2.0, P<0.05$ ) in the 2D group (Fig. 4C, Table S1). Most of these miRNAs recovered to the baseline levels in the 7D group, only eight up-regulated miRNAs and 11 down-regulated miRNAs were detected (Fold Change $\geq 2.0, \mathrm{P}<0.05$ ) (Fig. 4C, Table S2). The cumulative distribution frequency (CDF) plot showed that the top five up-regulated miRNAs (miR-148a-3p, miR-122-5p, miR-192-5p, miR-22-3p, and miR-21a-5p) in 2D group accounted for up to $84.27 \%$ of all detectable miRNAs (Fig. 4C). Compared with the CC group, only 13 miRNAs were up-regulated, and six miRNAs were down-regulated (fold change $\geq$ 2.0, $P<0.05$ ) in the $8 \mathrm{~W}$ group (Fig. 4D, Table S3); 8 miRNAs were up-regulated, and three miRNAs were down-regulated (fold change $\geq 2.0, \mathrm{P}<0.05$ ) in $12 \mathrm{~W}$ group (Fig. 4D, Table S4). The CDF analysis showed that the top three up-regulated miRNAs (miR-122-5p, miR-192-5p, and miR-22-3p) in 8W group constituted up to $43.48 \%$ of all detectable miRNAs (Fig S2). These findings suggested that both ALI and CLI induced changes in serum sEV miRNAs composition. The changes were caused by the differential expression of a small number of miRNAs with high abundance. 
The biological significance of these differentially expressed serum sEV miRNAs in liver injury was explored by Ingenuity Pathway Analysis (IPA, www.qiagen.com/ingenuity). As for the 176 differentially expressed serum sEV miRNAs upon ALI, hepatic steatosis was the most significant hepatotoxicity function, followed by liver inflammation. As for the 19 differentially expressed serum sEV miRNAs upon $\mathrm{CLI}$, hepatocellular carcinoma was the most significant, followed by liver hyperplasia (Fig. 4E).

\section{The liver is the main contributor to the differentially expressed serum sEV miRNAs during AlL and CLI.}

We were interested in know the contribution of liver cells to the change of serum sEV miRNAs upon ALI and CLI. Firstly, the liver miRNA expression profile for wild-type male C57BL/6 mice was established using BRB-Array Tool v4.6.0 (https://brb.nci.nih.gov) based on the RNA-seq data from GSE78792 (https://www.ncbi.nlm.nih.gov/geo/query/acc.cgi?acc=GSE78792) [17] (Table S5). Differentially expressed serum sEV miRNAs from ALI and CLI mice were compared to the liver miRNA expression profile. Among the top ten most abundant liver miRNAs, seven were the top ten increased miRNAs in serum sEVs upon ALI (Table 1) and constituted up to $84.70 \%$ of increased serum sEV miRNAs; as for CLI, three were raised in serum sEVs. These three miRNAs were exactly the top three miRNAs that increased upon CLI (Table 1) and constituted up to $60.56 \%$ of total increased serum sEV miRNAs. These findings suggested the liver as the primary contributor for the up-regulated serum sEV miRNAs during ALI and CLI and convinced that serum sEV miRNA test could be a reliable and sensitive way to monitor either acute or chronic liver injury. 
Table 1

Liver enriched miRNAs ranked high in serum SEV miRNAs from AIL and CIL model mice

\begin{tabular}{|c|c|c|c|c|c|c|c|}
\hline $\begin{array}{l}\text { GSE78792 } \\
\text { Liver }\end{array}$ & Rank & ALI & $\begin{array}{l}\text { ALI- } \\
\text { Rank }\end{array}$ & $\begin{array}{l}\text { GSE78792 } \\
\text { Liver }\end{array}$ & Rank & ALI & $\begin{array}{l}\text { ALI- } \\
\text { Rank }\end{array}$ \\
\hline miR-192 & 1 & $\begin{array}{l}\operatorname{miR}-192- \\
5 p\end{array}$ & 3 & miR-148a & 4 & $\begin{array}{l}\operatorname{miR-148a-} \\
3 p\end{array}$ & 1 \\
\hline miR-22 & 2 & miR-22-3p & 4 & miR-122 & 7 & $\begin{array}{l}\operatorname{miR}-122- \\
5 p\end{array}$ & 2 \\
\hline miR-30a & 3 & $\begin{array}{l}\text { miR-30a- } \\
5 p\end{array}$ & 7 & miR-192 & 1 & $\begin{array}{l}\operatorname{miR}-192- \\
5 p\end{array}$ & 3 \\
\hline miR-148a & 4 & $\begin{array}{l}\operatorname{miR-148a-} \\
3 p\end{array}$ & 1 & miR-22 & 2 & miR-22-3p & 4 \\
\hline miR-21a & 5 & $\begin{array}{l}\operatorname{miR}_{5 p}-21 a- \\
\end{array}$ & 5 & miR-21a & 5 & $\begin{array}{l}\operatorname{miR}-21 a- \\
5 p\end{array}$ & 5 \\
\hline miR-26a-2 & 6 & $\begin{array}{l}\operatorname{miR}-26 a- \\
5 p\end{array}$ & 105 & miR-30a & 3 & $\begin{array}{l}\operatorname{miR}-30 a- \\
5 p\end{array}$ & 7 \\
\hline miR-122 & 7 & $\begin{array}{l}\operatorname{miR}-122- \\
5 p\end{array}$ & 2 & miR-27b & 10 & $\begin{array}{l}\operatorname{miR}-27 b- \\
3 p\end{array}$ & 10 \\
\hline miR-10a & 8 & $\mathrm{n}$ & $\mathrm{n}$ & miR-143 & 9 & $\begin{array}{l}\operatorname{miR}-143- \\
3 p\end{array}$ & 92 \\
\hline miR-143 & 9 & $\begin{array}{l}\operatorname{miR}-143- \\
3 p\end{array}$ & 92 & miR-26a-2 & 6 & $\begin{array}{l}\operatorname{miR}_{5 p}-26 a- \\
\end{array}$ & 105 \\
\hline miR-27b & 10 & $\begin{array}{l}\operatorname{miR}-27 b- \\
3 p\end{array}$ & 10 & miR-10a & 8 & $\mathrm{n}$ & $n$ \\
\hline $\begin{array}{l}\text { GSE78792 } \\
\text { Liver }\end{array}$ & Rank & CLI & $\begin{array}{l}\text { CLI- } \\
\text { Rank }\end{array}$ & $\begin{array}{l}\text { GSE78792 } \\
\text { Liver }\end{array}$ & Rank & CLI & $\begin{array}{l}\text { CLI- } \\
\text { Rank }\end{array}$ \\
\hline miR-192 & 1 & $\begin{array}{l}\operatorname{miR}-192- \\
5 p\end{array}$ & 2 & miR-122 & 7 & $\begin{array}{l}\operatorname{miR}-122- \\
5 p\end{array}$ & 1 \\
\hline miR-22 & 2 & miR-22-3p & 3 & miR-192 & 1 & $\begin{array}{l}\operatorname{miR}-192- \\
5 p\end{array}$ & 2 \\
\hline miR-30a & 3 & $\mathrm{n}$ & $\mathrm{n}$ & miR-22 & 2 & miR-22-3p & 3 \\
\hline miR-148a & 4 & $\mathrm{n}$ & $\mathrm{n}$ & miR-148a & 4 & $\mathrm{n}$ & $\mathrm{n}$ \\
\hline miR-21a & 5 & $\mathrm{n}$ & $n$ & miR-21a & 5 & $\mathrm{n}$ & $n$ \\
\hline miR-26a-2 & 6 & $\mathrm{n}$ & $n$ & miR-30a & 3 & $\mathrm{n}$ & $n$ \\
\hline miR-122 & 7 & $\begin{array}{l}\operatorname{miR}_{5 p}-122- \\
\end{array}$ & 1 & miR-26a-2 & 6 & $\mathrm{n}$ & $\mathrm{n}$ \\
\hline
\end{tabular}

ALI, acute liver injury; CLI, chronic liver injury; n, not applicable. 


\begin{tabular}{|llllllll|}
\hline $\begin{array}{l}\text { GSE78792 } \\
\text { Liver }\end{array}$ & Rank & ALI & $\begin{array}{l}\text { ALI- } \\
\text { Rank }\end{array}$ & $\begin{array}{l}\text { GSE78792 } \\
\text { Liver }\end{array}$ & Rank & ALI & \begin{tabular}{l}
$\begin{array}{l}\text { ALI- } \\
\text { Rank }\end{array}$ \\
\hline miR-10a
\end{tabular} \\
\hline miR-143 & 9 & $\mathrm{n}$ & $\mathrm{n}$ & miR-10a & 8 & $\mathrm{n}$ & $\mathrm{n}$ \\
\hline miR-27b & 10 & $\mathrm{n}$ & $\mathrm{n}$ & miR-143 & 9 & $\mathrm{n}$ & $\mathrm{n}$ \\
\hline ALI, acute liver injury; CLI, chronic liver injury; n, not applicable. & & & $\mathrm{n}$ \\
\hline
\end{tabular}

\section{ALI and CLI signatures based on serum sEV miRNA profiling}

To identify serum sEV miRNA signatures for liver injury, we compared the differentially expressed serum sEV miRNAs in various stages of ALI and CLI. Compared to vehicle control, eight miRNAs were upregulated, and two miRNAs downregulated significantly during the acute injury stage and sustained through the chronic phase (Fold change $\geq 2.0, P<0.05$ ), which can serve as common liver injury signatures. Besides, 166 miRNAs changed significantly during acute injury, and nine miRNAs changed dramatically during chronic injury. These miRNAs with high abundance (TPM_Mean >1000), have the potential to be acute or chronic liver injury signatures. The complete lists of these potential ALI and CLI serum sEV miRNA signatures were provided in Table S6. According to their abundance, up to the top 20 miRNAs were listed in Table 2. For the recovery stage, 19 miRNAs changed significantly in the ALI group, 11 miRNAs changed significantly in the CLI group. Some of these miRNAs overlapped with differentially expressed serum sEV miRNAs in corresponding acute or chronic injury stages (Table 2). 
Table 2

Common and specific miRNAs for acute and chronic liver injury and recovery in mice model

\begin{tabular}{|c|c|c|c|c|c|}
\hline $\begin{array}{l}\text { ALI-Up } \\
\text { Specific }\end{array}$ & $\begin{array}{l}\text { CLI-Up } \\
\text { Specific }\end{array}$ & $\begin{array}{l}\text { Common- } \\
\text { Up }\end{array}$ & $\begin{array}{l}\text { ALI-Down } \\
\text { Specific }\end{array}$ & $\begin{array}{l}\text { CLI-Down } \\
\text { Specific }\end{array}$ & $\begin{array}{l}\text { Common- } \\
\text { Down }\end{array}$ \\
\hline miR-148a-3p & miR-409-3p & $\begin{array}{l}\operatorname{miR}_{5 p}-122- \\
\end{array}$ & miR-143-3p & miR-6239 & miR-5112 \\
\hline miR-21a-5p & miR-139-3p & $\begin{array}{l}\operatorname{miR}_{5 p}-192- \\
\end{array}$ & miR-451a & miR-8109 & miR-9-5p \\
\hline miR-92a-3p & miR-690 & miR-22-3p & miR-206-3p & miR-10b-3p & \\
\hline miR-30a-5p & miR-211-5p & miR-22-5p & let-7c-5p & miR-8117 & \\
\hline miR-140-3p & miR-1941-5p & $\begin{array}{l}\text { miR-708- } \\
3 p\end{array}$ & miR-128-3p & & \\
\hline miR-29a-3p & & $\begin{array}{l}\operatorname{miR-193a-} \\
5 p\end{array}$ & let-7b-5p & & \\
\hline miR-27b-3p & & $\begin{array}{l}\operatorname{miR}-344 d- \\
3 p\end{array}$ & miR-215-5p & & \\
\hline miR-101b-3p & & $\begin{array}{l}\operatorname{miR}-193 a- \\
3 p\end{array}$ & miR-27a-3p & & \\
\hline miR-423-3p & & & miR-184-3p & & \\
\hline miR-194-5p & & & miR-423-5p & & \\
\hline miR-30a-3p & & & miR-126a-3p & & \\
\hline miR-1839-5p & & & miR-5106 & & \\
\hline miR-152-3p & & & miR-26a-5p & & \\
\hline let-7d-3p & & & miR-142a-5p & & \\
\hline miR-7a-5p & & & miR-181a-5p & & \\
\hline miR-221-3p & & & miR-150-5p & & \\
\hline miR-203-3p & & & miR-200a-3p & & \\
\hline miR-185-5p & & & miR-100-5p & & \\
\hline miR-3535 & & & miR-375-3p & & \\
\hline miR-28a-3p & & & miR-139-5p & & \\
\hline $\begin{array}{l}\text { ALI-R-Up } \\
\text { Specific }\end{array}$ & $\begin{array}{l}\text { CLI-R-Up } \\
\text { Specific }\end{array}$ & $\begin{array}{l}\text { Common- } \\
\text { Up }\end{array}$ & $\begin{array}{l}\text { ALI-R-Down } \\
\text { Specific }\end{array}$ & $\begin{array}{l}\text { CLI-R-Down } \\
\text { Specific }\end{array}$ & $\begin{array}{l}\text { Common } \\
\text { Down }\end{array}$ \\
\hline
\end{tabular}

ALI, acute liver injury stage, CLI, chronic liver injury stage, ALI-R, acute liver injury-recovery stage, CLI-R, chronic liver injury-recovery stage, Fold change $\geq 2.0, P<0.05$; the bold font, TPM_Mean > 1000; the blue font, overlapping miRNAs in injury and recovery stages. 


\begin{tabular}{|c|c|c|c|c|c|}
\hline $\begin{array}{l}\text { ALI-Up } \\
\text { Specific }\end{array}$ & $\begin{array}{l}\text { CLI-Up } \\
\text { Specific }\end{array}$ & $\begin{array}{l}\text { Common- } \\
\text { Up }\end{array}$ & $\begin{array}{l}\text { ALI-Down } \\
\text { Specific }\end{array}$ & $\begin{array}{l}\text { CLI-Down } \\
\text { Specific }\end{array}$ & $\begin{array}{l}\text { Common- } \\
\text { Down }\end{array}$ \\
\hline miR-146b-5p & miR-122-5p & & miR-292a-5p & miR-6239 & \\
\hline miR-223-5p & miR-26b-5p & & miR-6538 & miR-8109 & \\
\hline miR-27a-5p & miR-142a-3p & & miR-374b-5p & miR-7689-3p & \\
\hline miR-147-3p & miR-103-3p & & miR-503-5p & & \\
\hline miR-615-3p & miR-374b-5p & & miR-7b-5p & & \\
\hline miR-1943-5p & miR-211-5p & & miR-450b-5p & & \\
\hline \multirow{5}{*}{$\begin{array}{l}\operatorname{miR}-181 a-2- \\
3 p\end{array}$} & miR-337-5p & & miR-431-3p & & \\
\hline & miR-1941-5p & & miR-431-5p & & \\
\hline & & & miR-292a-3p & & \\
\hline & & & miR-450b-3p & & \\
\hline & & & miR-181c-3p & & \\
\hline
\end{tabular}

\section{Comparison of the potential ALI and CLI serum sEV miRNA signatures to human liver diseases (HLD)- related circulating miRNAs}

To explore the biological significance of the ALI and CLI serum sEV miRNA signatures in HLDs, we performed a systemic review of abnormally expressed circulating miRNAs reported in various HLDs. A total of 299 and 257 studies were identified from Pubmed (https://pubmed.ncbi.nlm.nih.gov ) and WOS (http://apps.webofknowledge.com/) database, respectively (Fig. 5A). Data were retrieved from 14 studies, including drug-induced liver injury (DILI) [18], chronic hepatitis B (CHB) [19-23], chronic hepatitis C (CHC) [19, 23-25], non-alcoholic fatty liver disease (NAFLD) [26], non-alcoholic steatohepatitis (NASH) [23], liver cirrhosis (LC) $[22,27,28]$ and HCC [19-22, 26-31]. Details of the 14 articles were summarized in Table S7.

Totally 269 abnormally expressed circulating miRNAs related to HLDs were extracted, which appeared $\geq$ four times were defined as high frequency (Fig. 5A). Of the 30 high-frequency miRNAs (Table S7), 23 were detected in ALI and CLI serum sEVs (Fig. 5B), 14 miRNAs overlapped with ALI and CLI serum sEV signatures, and 12 miRNAs showed the same expression trend. Of the 12 miRNAs, three miRNAs (miR122-5p, miR-192-5p, and miR-22-3p) were identified as common injury signatures that increased in both $\mathrm{ALI}$ and CLI mice and thus have the potential to serve as common signatures for either acute or chronic liver injury. The other 9 miRNAs were identified as ALI signatures (miR-21a-5p, miR-92a-3p, miR-194-5p, 
miR-17-5p and miR-19b-3p increased, and miR-451a, miR-27a-3p, miR-26a-5p, and miR-223-3p decreased), and may reflect acute or active liver injury (Fig. 5C).

\section{Serum sEVs from liver injury mice induced hepatic macrophages reprogramming}

Primary mouse hepatic macrophages were isolated, purified and incubated with SYTO labeled serum sEVs. After $24 \mathrm{~h}$, green fluorescence can be observed in most macrophages in both control and ALI (2D) serum sEVs incubation groups (Fig. 6A). These observations proved that serum sEVs could be uptaken by hepatic macrophages. Serum sEVs from ALI mice (2D) could accelerate the adhesion of hepatic macrophages (Fig. 6B). As determined by qRT-PCR, normal serum sEVs increased the M1-like gene (IL-1B and TNFA) expression, and decrease some of the M2-like gene (IL10RB, CD163, ARG1, and CD206) expression; ALI serum sEVs showed similar effects. However, compared to normal serum sEVs, ALI serum sEVs tended to decrease M1-like IL-1B but increase the M2-like CD163 gene expression (Fig. 6C).

To further dissect the effects of ALI serum sEVs on monocyte-derived and resident hepatic macrophage subgroups, multiple-color flow cytometric analyses were performed to access the expression of M1-like CD86 and the M2-like CD206 in CD11 b ${ }^{+} \mathrm{F} 4 / 80^{\text {Low }}$ monocyte-derived and CD11b ${ }^{+} \mathrm{F} 4 / 80^{\text {High }}$ resident macrophages ([32] and our unpublished data). It turned out that ALI serum sEVs decreased both CD86 and CD206 expression in CD11 $\mathrm{b}^{+} \mathrm{F} 4 / 80^{\mathrm{Low}}$ subgroup, but increased CD206 expression in $\mathrm{CD} 11 \mathrm{~b}^{+} \mathrm{F} 4 / 80^{\mathrm{High}}$ subgroup (Fig. $6 \mathrm{D}-\mathrm{E}$ ). These findings indicated that ALI serum sEVs might induce depolarization of $\mathrm{CD} 11 \mathrm{~b}^{+} \mathrm{F} 4 / 80^{\text {Low }}$ monocyte-derived macrophages, but M2 differentiation of $\mathrm{CD} 11 \mathrm{~b}^{+} \mathrm{F} 4 / 80^{\text {High }}$ resident macrophages.

\section{Discussion}

A growing number of studies suggested the diagnostic value of serum sEV contents for liver injury, especially miRNAs $[6,8]$. To explore the potential serum sEV miRNA biomarkers for liver injury, we simulated the complex process of liver injury and recovery by $\mathrm{CCL}_{4}$ induced acute and chronic liver injury mice models.

Unexpectedly, aside from differentially expressed miRNAs, we found that the concentration, size, and morphology of serum sEVs might be essential features in liver injury (Fig. 2). The number of serum sEVs increased upon ALI, which has been reported in human alcoholic hepatitis and alcoholic liver injury mice model [33-35]. Furthermore, we found a sustained decrease in serum sEVs number during the chronic injury stage and the recovery stage for both acute and chronic liver injury. What's more, serum sEVs became smaller upon ALI, but enlarged during ALI recovery and CLI stage, and persisted through CLI recovery stage, coupled with multiple peaks as observed by NTA. Interestingly, the aggregation of serum sEV particles was observed in samples from the ALI recovery stage, CLI stage, and CLI recovery stage by TEM, which explained the multiple peaks and increased particle size found by NTA. We proposed that the aggregation of $s E V s$ might reflect the membrane damage of extracellular vesicles following liver injury. Thus, the changes in serum sEVs concentration, size, and morphology were well connected to a particular 
stage of liver injury and could provide diagnostic clues. Besides, the changes of serum sEVs persisted even when the liver function tests and visible histopathological changes were restored, which could be useful to trace recent liver injury.

Then we showed that ALI and CLI altered the sRNA contents and components in serum sEVs. For ALI, both total RNA and sRNA contents of serum sEVs increased significantly, coupled with an increased proportion of miRNAs in sRNA. Although there was no significant increase in RNA and sRNAs for CLI, the proportion of miRNAs increased significantly. Compare to CLI, more miRNAs species changed significantly upon ALI (176 vs. 19, fold change $\geq 2.0, \mathrm{P}<0.05$ ). And the increased proportion of miRNAs upon liver injury was mainly contributed by a few high-abundant miRNAs. To traceback the primary source of the increased serum sEV miRNAs, the high-abundant miRNAs detected in serum sEVs from ALI and CLI mice were evaluated in the livers of normal male C57BL/ 6 mice. It turned out that the miRNAs of the highest abundance were all liver enriched miRNAs (Table 2). We proposed that the liver was the main contributor to the elevated serum sEV-miRNAs for both acute and chronic liver injury. Thus, serum sEVs carried the miRNA messages released from the injured liver, examing the serum sEV miRNAs could be a reliable way to monitor either acute or chronic liver injury.

By comparing the expression profiles of serum sEV miRNAs in various stages of ALI and CLI, we obtained a list of miRNAs as common liver injury signatures, as well as the miRNA signatures for ALI, CLI, and the recovering stages. However, these signatures were obtained from mice models and needed to be validated in human patients. Hence, we carried out a systemic review of previously published studies and obtained 30 miRNAs that were highly correlated with human liver disease, including DILI, CHB, CHC, NAFLD, NASH, and liver cirrhosis (Fig. 5). Of these 30 miRNAs, three miRNAs (miR-122-5p, miR-192-5p, and miR-22-3p) were identified as common injury signatures that increased in both ALI and CLI mice. Although these three miRNAs have been studied separately [22, 34, 36, 37], here for the first time, they were combined as a universal signature for either acute or chronic liver injury. Nine miRNAs were identified as ALI signatures. Along with the five increasing miRNAs (miR-21a-5p, miR-92a-3p, miR-194-5p, miR-17-5p, and miR-19b-3p), four miRNAs decreased (miR-451a, miR-27a-3p, miR-26a-5p, and miR-223$3 p)$, which were exactly the four decreased miRNAs that frequently reported in human liver disease (Fig. 5). Although the human data were mostly from patients with chronic liver injury, the overlapping of ALI signatures might reflect active lesions. Based on serum sEV miRNAs, we established common signatures for liver injury and specific ones for acute/active liver injury.

The biological significance of the altered sEV miRNAs upon liver injury were explored by IPA analyses. The top hepatotoxicity functions of the altered serum sEVs miRNAs in ALI were hepatic steatosis and liver inflammation, while the altered miRNAs carried by serum sEVs from CLI were linked to hepatocellular carcinoma, followed by liver hyperplasia. These findings were well consistent with the clinical facts that ALI mainly caused inflammation, while sustained damage increased the risk of carcinoma [38, 39]. Hepatic steatosis reflected fatty degeneration that was typically caused by $\mathrm{CCL}_{4}$ [40]. These findings revealed the pathological importance of serum sEVs during the process of acute and chronic liver injury. 
It has been reported that hepatic macrophages can take up serum sEVs, and play essential roles in the clearance of intravenously injected sEVs from the systemic circulation [41, 42]. We were interested in knowing the effects of the liver injury serum sEVs on hepatic macrophages and if these effects could aggravate the liver damage or play a pprotective role. We found in vitro, ALI serum sEVs could be uptaken by hepatic macrophages and promote their adhesion. Furthermore, ALI serum sEVs tended to decrease the M1 like gene expressions, such as IL-1B and TNFA, and increase the M2 like gene expression, including CD163. But the trends were not consistent. Considering that hepatic macrophages are heterogeneous populations composed of two subgroups, including resident macrophages and circulating monocyte-derived macrophages [32], we proposed macrophages of different origins might react inconsistently to ALI serum sEVs. The expression of M1 (CD86) and M2 (CD206) polarization signature on the two subgroups of hepatic macrophages were examined by flow cytometry. It turned out that ALI serum $\mathrm{SEV}$ s induced depolarization of $\mathrm{CD} 11 \mathrm{~b}^{+} \mathrm{F} 4 / 80^{\mathrm{Low}}$ monocyte-derived macrophages, but induced $\mathrm{M} 2$ differentiation of $\mathrm{CD} 11 \mathrm{~b}^{+} \mathrm{F} 4 / 80^{\mathrm{High}}$ resident macrophages. We proposed that the changes of serum sEVs upon ALI might alleviate liver damage by depolarizing monocyte-derived macrophages and educating resident hepatic macrophage to $\mathrm{M} 2$ like cells.

\section{Conclusion}

In conclusion, we showed that the concentration, size, and morphology of serum sEV particles were essential features for liver injury. Specific serum sEV miRNA signatures for different liver injury stages and a list of miRNAs as common liver injury biomarkers were established. The altered ALI and CLI serum sEVs miRNAs were connected to diverse liver pathological processes. The ALI serum sEVs reprogrammed hepatic macrophage subgroups differently. Serum sEVs not only have good diagnostic potential but also could be used for the cure of liver injury. However, the diagnostic and therapeutic potential of these altered serum sEVs upon liver injury deserved further study.

\section{Abbreviations}

sEVs, small Extracellular vesicles; ALI, acute liver injury; CLI, chronic liver injury; ALT, alanine aminotransferases; $\mathrm{AST}$, aspartate aminotransferase; $\mathrm{HCC}$, hepatocellular carcinoma; $\mathrm{CCL}_{4}$, carbon tetrachloride; TEM, transmission electron microscopy; NTA, nanoparticle tracking analysis; RNA-seq, RNA sequencing; TPM, transcripts per million reads; IPA, Ingenuity Pathway; HLD, human liver disease; DILI, drug-induced liver injury; $\mathrm{CHB}$, chronic hepatitis $\mathrm{B} ; \mathrm{CHC}$, chronic hepatitis C; NAFLD, non-alcoholic fatty liver disease; NASH, non-alcoholic steatohepatitis; LC, liver cirrhosis.

\section{Declarations}

\section{Ethics approval and consent to participate}

All experimental protocols were approved by the Animal Ethics Committee of Nantong University. Animal care and experiments were performed in line with the relevant guidelines and regulations. 


\section{Consent for publication}

Not applicable.

\section{Availability of data and materials}

All data included in the present study were presented in the main manuscript and its supplementary information files.

\section{Competing of interest}

The authors declared that no competing of interest.

\section{Funding}

The study was supported by the National Natural Science Foundation of China (NSFC) grants to J.J. (No. 81761128018 , No. 81572871) and Y.J. (No. 81272027), and by the Natural Science Foundation of Jiangsu Province grant to J.J. (No. BK20151277). The funders had no role in study design, data collection, and analysis, decision to publish, or preparation of the manuscript.

\section{Author contributions}

J.J. and Y.J. conceived the study and interpreted the data; X.L. performed a systemic review and statistical analysis; J.L., L.H., and P.L. performed the mice model experiments; L.C. performed the mice model and the primary mouse hepatic macrophage related experiments; M.Z. isolated the serum sEVs and performed the characterization; Y.S. performed the histopathologic examination and the SEVs characterization; W.L. performed qRT-PCR; A.Z. performed the western blot; G.Q. performed the liver function test; J.J. and X.L. prepared the figures and drafted the manuscript. All authors read and approved the final version of the manuscript.

\section{Acknowledgements}

Not applicable

\section{Authors' information}

1 Department of Pathology, Medical School of Nantong University, Nantong, 226001, China. 2 Key Laboratory of Microenvironment and Translational Cancer Research, Nantong, 226001, China. 3 Department of Pathology, National Cancer Center/National Clinical Research Center for Cancer/Cancer Hospital, Chinese Academy of Medical Sciences and Peking Union Medical College, Beijing, 100021, China. 4 Department of Pathology, Nanjing Jinling Hospital, Nanjing University School of Medicine, Nanjing, 210093, China. 5 Department of Pathology, Affiliated Jiangyin Hospital of Southeast University Medical College, Jiangyin, 214400, China. 6 Center for Liver Diseases, the Third Affiliated Hospital of Nantong University, Nantong, 226006, China. 7 Key Laboratory of Neuroregeneration of Jiangsu and 
Ministry of Education, Nantong University, Nantong, 226001, China. 8 Institute of Immunology, College of Life Science and Technology, Jinan University, Guangdong, 510632, China.

\section{References}

1. Reuben A, Koch DG, Lee WM, Acute Liver Failure Study G. Drug-induced acute liver failure: results of a U.S. multicenter, prospective study. Hepatology 2010; 52:2065-2076.

2. Kullak-Ublick GA, Andrade RJ, Merz M, End P, Benesic A, Gerbes AL, Aithal GP. Drug-induced liver injury: recent advances in diagnosis and risk assessment. Gut 2017; 66:1154-1164.

3. Nathwani RA, Pais S, Reynolds TB, Kaplowitz N. Serum alanine aminotransferase in skeletal muscle diseases. Hepatology 2005; 41:380-382.

4. Hubbell CL, Mankes RF, Reid LD. A small dose of morphine leads rats to drink more alcohol and achieve higher blood alcohol concentrations. Alcohol Clin Exp Res 1993; 17:1040-1043.

5. Théry C, Witwer KW, Aikawa E, Alcaraz MJ, Anderson JD, Andriantsitohaina R, Antoniou A, Arab T, Archer F, Atkin-Smith GK, et al. Minimal information for studies of extracellular vesicles 2018 (MISEV2018): a position statement of the International Society for Extracellular Vesicles and update of the MISEV2014 guidelines. J Extracell Vesicles 2018; 7:1535750.

6. Szabo G, Momen-Heravi F. Extracellular vesicles in liver disease and potential as biomarkers and therapeutic targets. Nat Rev Gastroenterol Hepatol 2017; 14:455-466.

7. Valadi H, Ekstrom K, Bossios A, Sjostrand M, Lee JJ, Lotvall JO. Exosome-mediated transfer of mRNAs and microRNAs is a novel mechanism of genetic exchange between cells. Nature Cell Biology 2007; 9:654-U672.

8. Urban SK, Mocan T, Sanger H, Lukacs-Kornek V, Kornek M. Extracellular Vesicles in Liver Diseases: Diagnostic, Prognostic, and Therapeutic Application. Semin Liver Dis 2019; 39:70-77.

9. Bartel DP. MicroRNAs: genomics, biogenesis, mechanism, and function. Cell 2004; 116:281-297.

10. Erdos Z, Barnum JE, Wang E, DeMaula C, Dey PM, Forest T, Bailey WJ, Glaab WE. Evaluation of the Relative Performance of Pancreas-Specific MicroRNAs in Rat Plasma as Biomarkers of Pancreas Injury. Toxicol Sci 2020; 173:5-18.

11. Laterza OF, Lim L, Garrett-Engele PW, Vlasakova K, Muniappa N, Tanaka WK, Johnson JM, Sina JF, Fare TL, Sistare FD, Glaab WE. Plasma MicroRNAs as sensitive and specific biomarkers of tissue injury. Clin Chem 2009; 55:1977-1983.

12. Sun P, Liu DZ, Jickling GC, Sharp FR, Yin KJ. MicroRNA-based therapeutics in central nervous system injuries. J Cereb Blood Flow Metab 2018; 38:1125-1148.

13. Wang XW, Heegaard NHH, Orum H. MicroRNAs in Liver Disease. Gastroenterology 2012; 142:14311443.

14. Cheng L, Sharples RA, Scicluna BJ, Hill AF. Exosomes provide a protective and enriched source of miRNA for biomarker profiling compared to intracellular and cell-free blood. J Extracell Vesicles 2014; 3. 
15. Gu L, Deng WS, Sun XF, Zhou H, Xu Q. Rapamycin ameliorates CCl4-induced liver fibrosis in mice through reciprocal regulation of the Th17/Treg cell balance. Mol Med Rep 2016; 14:1153-1161.

16. Zhao F, Cheng L, Shao Q, Chen Z, Lv X, Li J, He L, Sun Y, Ji Q, Lu P, et al. Characterization of serum small extracellular vesicles and their small RNA contents across humans, rats, and mice. Scientific Reports 2020; 10:4197.

17. Langfelder P, Gao F, Wang N, Howland D, Kwak S, Vogt TF, Aaronson JS, Rosinski J, Coppola G, Horvath S, Yang XW. MicroRNA signatures of endogenous Huntingtin CAG repeat expansion in mice. PLoS One 2018; 13:e0190550.

18. Russo MW, Steuerwald N, Norton HJ, Anderson WE, Foureau D, Chalasani N, Fontana RJ, Watkins PB, Serrano J, Bonkovsky HL. Profiles of miRNAs in serum in severe acute drug induced liver injury and their prognostic significance. Liver Int 2017; 37:757-764.

19. Li LM, Hu ZB, Zhou ZX, Chen X, Liu FY, Zhang JF, Shen HB, Zhang CY, Zen K. Serum microRNA profiles serve as novel biomarkers for HBV infection and diagnosis of HBV-positive hepatocarcinoma. Cancer Res 2010; 70:9798-9807.

20. Wang Y, Gao Y, Shi W, Zhai D, Rao Q, Jia X, Liu J, Jiao X, Du Z. Profiles of differential expression of circulating microRNAs in hepatitis $B$ virus-positive small hepatocellular carcinoma. Cancer Biomark 2015; 15:171-180.

21. Sohn W, Kim J, Kang SH, Yang SR, Cho JY, Cho HC, Shim SG, Paik YH. Serum exosomal microRNAs as novel biomarkers for hepatocellular carcinoma. Exp Mol Med 2015; 47:e184.

22. Zhou J, Yu L, Gao X, Hu J, Wang J, Dai Z, Wang J-F, Zhang Z, Lu S, Huang X, et al. Plasma MicroRNA Panel to Diagnose Hepatitis B Virus-Related Hepatocellular Carcinoma. Journal of Clinical Oncology $2011 ; 29: 4781-4788$.

23. Murakami Y, Toyoda H, Tanahashi T, Tanaka J, Kumada T, Yoshioka Y, Kosaka N, Ochiya T, Taguchi Yh. Comprehensive miRNA Expression Analysis in Peripheral Blood Can Diagnose Liver Disease. Plos One 2012; 7.

24. Murakami Y, Tanahashi T. Analysis of circulating microRNA by microarray in liver disease. Methods Mol Biol 2013; 1024:173-182.

25. Santangelo L, Bordoni V, Montaldo C, Cimini E, Zingoni A, Battistelli C, D'Offizi G, Capobianchi MR, Santoni A, Tripodi M, Agrati C. Hepatitis C virus direct-acting antivirals therapy impacts on extracellular vesicles microRNAs content and on their immunomodulating properties. Liver Int 2018; 38:1741-1750.

26. Tan Y, Ge G, Pan T, Wen D, Gan J. A pilot study of serum microRNAs panel as potential biomarkers for diagnosis of nonalcoholic fatty liver disease. PLoS One 2014; 9:e105192.

27. Gui J, Tian Y, Wen X, Zhang W, Zhang P, Gao J, Run W, Tian L, Jia X, Gao Y. Serum microRNA characterization identifies miR-885-5p as a potential marker for detecting liver pathologies. Clin Sci (Lond) $2011 ; 120: 183-193$.

28. Fornari F, Ferracin M, Trere D, Milazzo M, Marinelli S, Galassi M, Venerandi L, Pollutri D, Patrizi C, Borghi A, et al. Circulating microRNAs, miR-939, miR-595, miR-519d and miR-494, Identify Cirrhotic 
Patients with HCC. PLoS One 2015; 10:e0141448.

29. Chiu LY, Kishnani PS, Chuang TP, Tang CY, Liu CY, Bali D, Koeberl D, Austin S, Boyette K, Weinstein DA, et al. Identification of differentially expressed microRNAs in human hepatocellular adenoma associated with type I glycogen storage disease: a potential utility as biomarkers. J Gastroenterol 2014; 49:1274-1284.

30. Lu L, Guo D, Chen X, Xiong W, Jie S, Li H. Abnormal miRNAs Targeting Chromosome Open Reading Frame Genes were Enriched in Microvesicles Derived from the Circulation of HCC. Biochem Genet 2016; 54:120-133.

31. Xue X, Zhao Y, Wang X, Qin L, Hu R. Development and validation of serum exosomal microRNAs as diagnostic and prognostic biomarkers for hepatocellular carcinoma. J Cell Biochem 2019; 120:135142.

32. Tacke F, Zimmermann HW. Macrophage heterogeneity in liver injury and fibrosis. Journal of hepatology 2014; 60:1090-1096.

33. Momen-Heravi F, Saha B, Kodys K, Catalano D, Satishchandran A, Szabo G. Increased number of circulating exosomes and their microRNA cargos are potential novel biomarkers in alcoholic hepatitis. Journal of Translational Medicine 2015; 13.

34. Momen-Heravi F, Bala S, Kodys K, Szabo G. Exosomes derived from alcohol-treated hepatocytes horizontally transfer liver specific miRNA-122 and sensitize monocytes to LPS. Sci Rep 2015; 5:9991.

35. Eguchi A, Lazaro RG, Wang J, Kim J, Povero D, Willliams B, Ho SB, Stärkel P, Schnabl B, OhnoMachado $L$, et al. Extracellular vesicles released by hepatocytes from gastric infusion model of alcoholic liver disease contain a MicroRNA barcode that can be detected in blood. Hepatology 2017; 65:475-490.

36. Motawi TK, Mohamed MR, Shahin NN, Ali MAM, Azzam MA. Time-course expression profile and diagnostic potential of a miRNA panel in exosomes and total serum in acute liver injury. The International Journal of Biochemistry \& Cell Biology 2018; 100:11-21.

37. Arataki K, Hayes CN, Akamatsu S, Akiyama R, Abe H, Tsuge M, Miki D, Ochi H, Hiraga N, Imamura M, et al. Circulating microRNA-22 correlates with microRNA-122 and represents viral replication and liver injury in patients with chronic hepatitis B. Journal of Medical Virology 2013; 85:789-798.

38. Michelotti GA, Machado MV, Diehl AM. NAFLD, NASH and liver cancer. Nat Rev Gastroenterol Hepatol $2013 ; 10: 656-665$.

39. Nguyen VT, Law MG, Dore GJ. Hepatitis B-related hepatocellular carcinoma: epidemiological characteristics and disease burden. J Viral Hepat 2009; 16:453-463.

40. Recknagel RO, Glende EA, Jr., Dolak JA, Waller RL. Mechanisms of carbon tetrachloride toxicity. Pharmacology \& therapeutics 1989; 43:139-154.

41. Imai T, Takahashi Y, Nishikawa M, Kato K, Morishita M, Yamashita T, Matsumoto A, Charoenviriyakul C, Takakura Y. Macrophage-dependent clearance of systemically administered B16BL6-derived exosomes from the blood circulation in mice. J Extracell Vesicles 2015; 4:26238. 
42. Matsumoto A, Takahashi Y, Chang HY, Wu YW, Yamamoto A, Ishihama Y, Takakura Y. Blood concentrations of small extracellular vesicles are determined by a balance between abundant secretion and rapid clearance. J Extracell Vesicles 2020; 9:1696517.

\section{Figures}

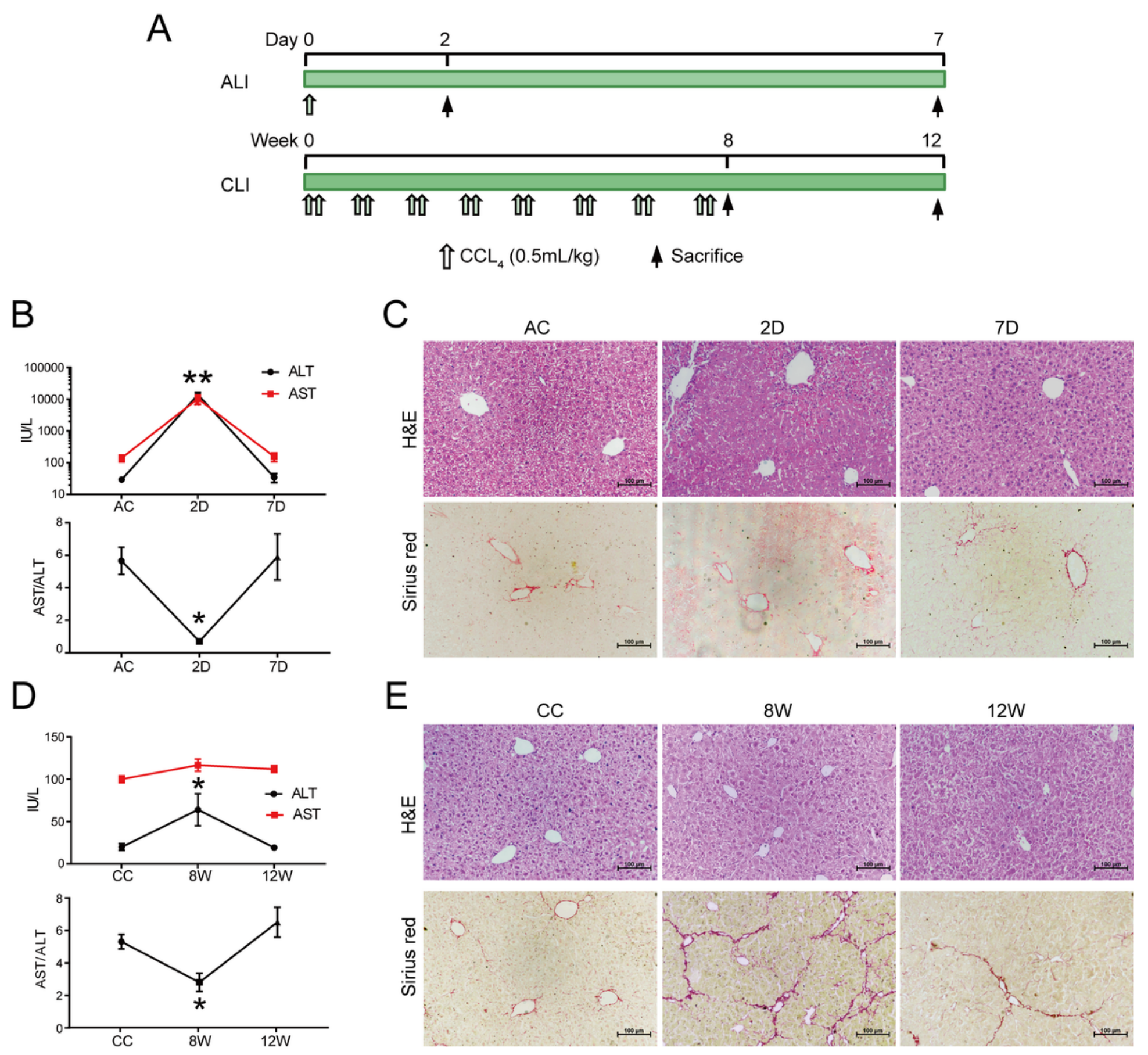

Figure 1

Establishment and validation of CCL4-induced acute liver injury (ALI) and chronic liver injury (CLI) in mice. (A) Workflow for the establishment of ALI and CLI mice model. (B) The serum ALT and AST levels in 
ALI mice. Compared with AC group, $* P<0.05,{ }^{*} P<<0.01$. (C) H\&E and Sirius red staining of liver sections from AC, 2D, and 7D mice. (D) The serum ALT and AST levels in CLI mice. Compared with CC group, *P < 0.05. (E) H\&E and Sirius red staining of liver sections from CC, 8W, and 12W mice. Scale bar $=100 \mu \mathrm{m}$. D, day; W, week; AC, control for ALI; CC, control for CLI.

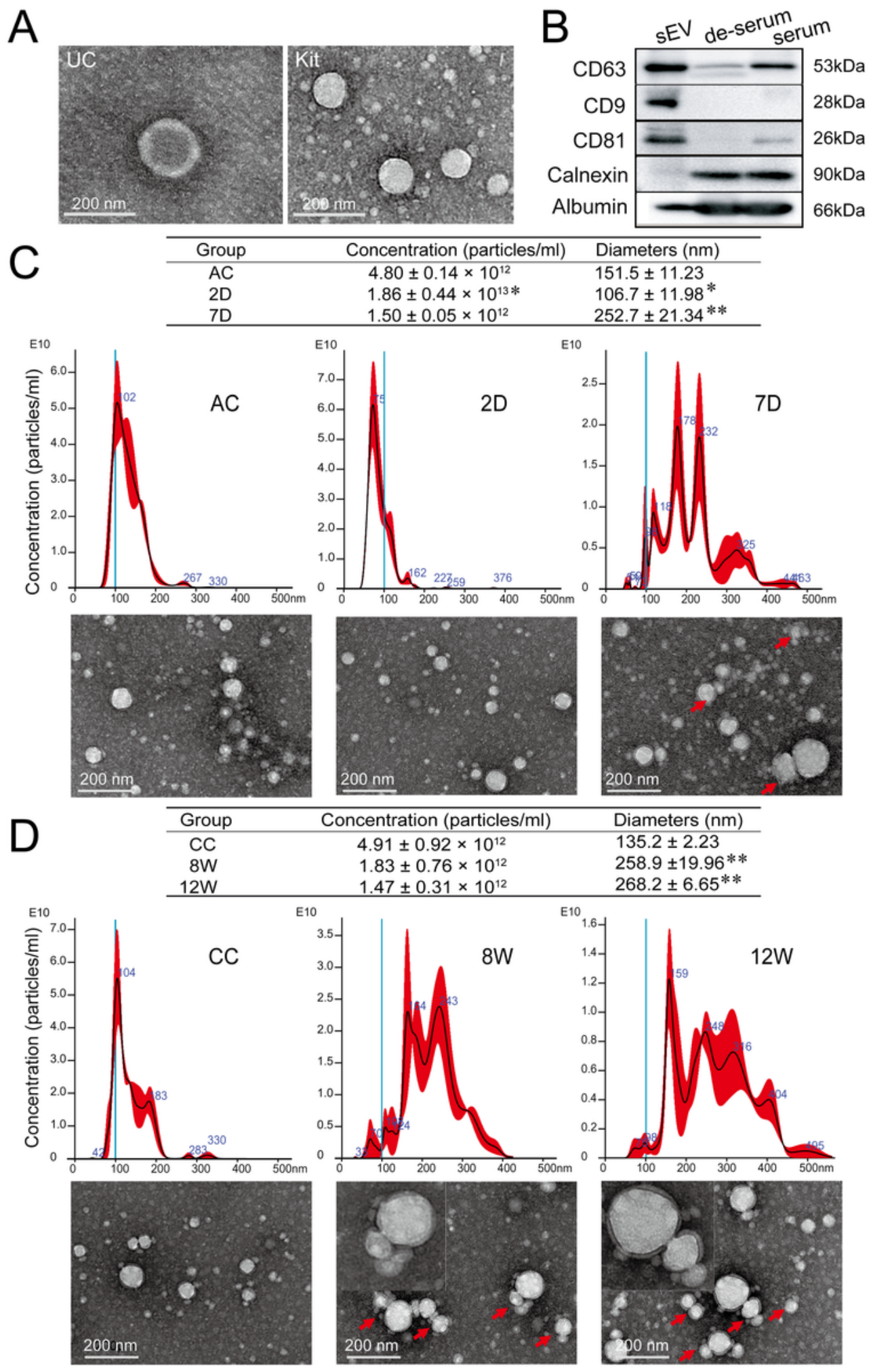

Figure 2 
Characterization of isolated mice serum small exracellular vesicle (sEV). (A) The TEM images of the isolated particles using ultracentrifugation and ExoQuick precipitation kit. (B) Representative western blotting bands for CD63, CD81, CD9, Calnexin, and Albumin. (C) The NTA plots for the size distribution and concentration of the isolated particles in each group from ALI mice and the corresponding TEM images. Compared with AC group, ${ }^{*} P<0.05,{ }^{*} P<0.01$. (D) The NTA plots for the size distribution and concentration of the isolated particles in each group from CLI mice, and the corresponding TEM images. Compared with CC group, ${ }^{\star \star} \mathrm{P}<0.01$. The red arrow indicates aggregated particles; representative particles were amplified and exemplified in the top left corner. Scale bar $=200 \mathrm{~nm}$. UC, ultra-centrifuge; TEM, transmission electron microscopy; D, day; W, week; AC, control for ALI; CC, control for CLI. 
A

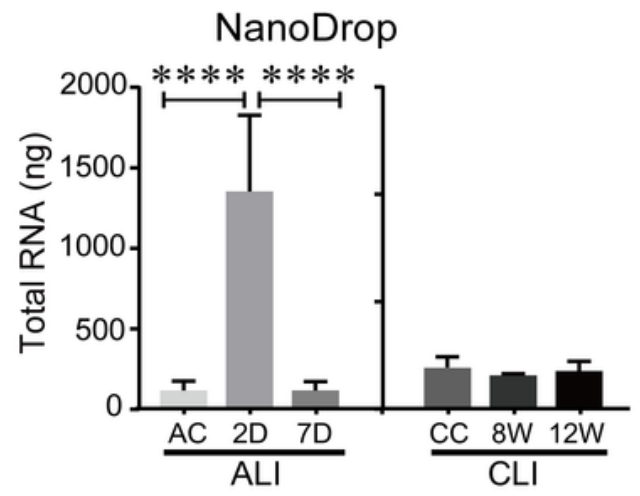

B

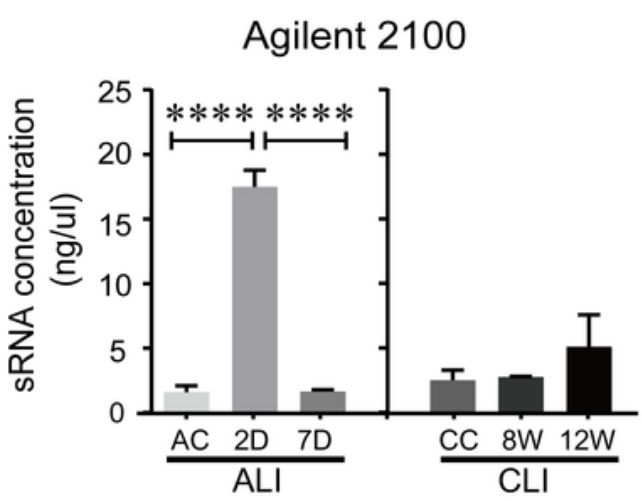

2D

7D
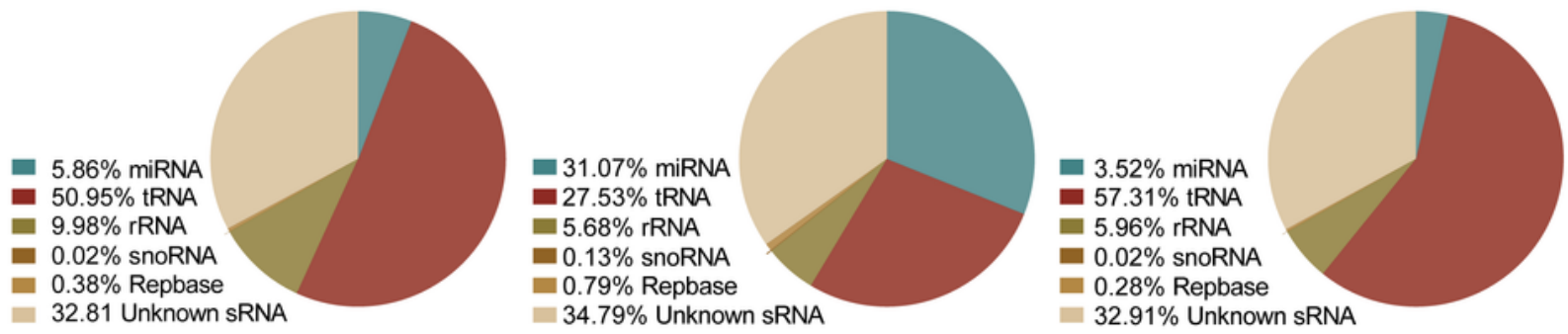

$\mathrm{CC}$

$8 W$

$12 \mathrm{~W}$
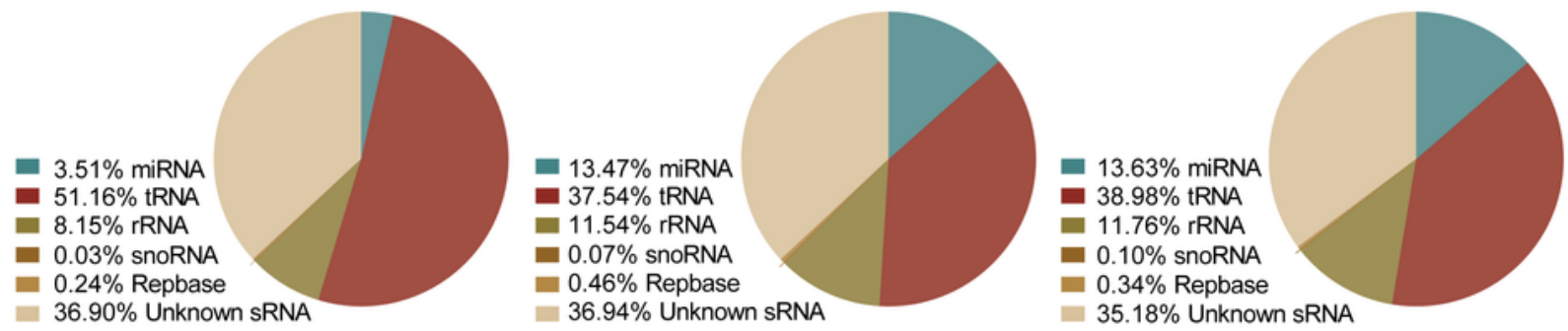

D

ALI
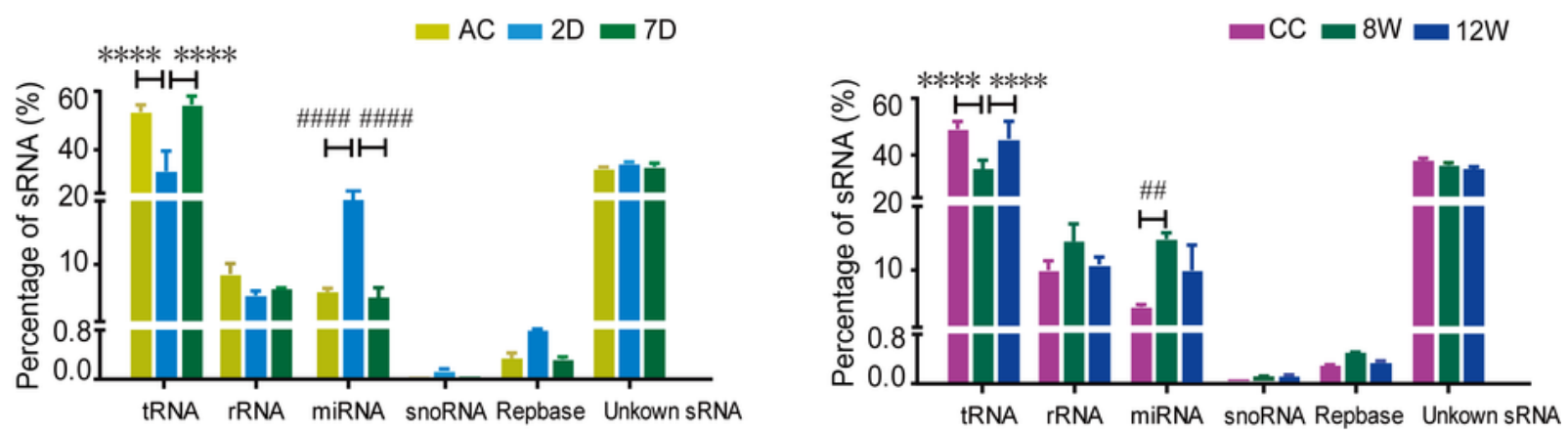

\section{Figure 3}

Annotations of sRNA in serum sEVs from ALI and CLI mice. (A) The total RNAs in serum sEVs, compared with the $2 \mathrm{D}$ group, $\star \star \star P<0.001$. (B) The sRNA concentration from serum $s E V s$, compared with the 2D group, ${ }^{* \star} P<0.001$. (C) Representative pie charts depict annotated sRNA species and their proportions in each group. (D) The statistical analysis of sRNA species in sEVs, compared with 2D group, ${ }^{\star \star \star \star *} \mathrm{P}<$ 
0.0001; compared with 8W group, \#\#P < 0.01, \#\#\#\# < 0.0001. D, day; W, week; AC, control for ALl; CC, control for CLI.

A

$\begin{array}{llllll}A C-1 & A C-2 & 2 D-1 & 2 D-2 & 7 D-1 & 7 D-2\end{array}$

$\begin{array}{lllllllll}\text { AC-1 } & 1.000 & 0.970 & 0.477 & 0.329 & 0.895 & 0.865\end{array}$

$\begin{array}{lllllll}\text { AC-2 } & 0.970 & 1.000 & 0.507 & 0.361 & 0.898 & 0.872\end{array}$

$\begin{array}{lllllll}\text { 2D-1 } & 0.477 & 0.507 & 1.000 & 0.963 & 0.680 & 0.713\end{array}$

$\begin{array}{lllllll}2 \mathrm{D}-2 & 0.329 & 0.361 & 0.963 & 1.000 & 0.542 & 0.579\end{array}$

$\begin{array}{lllllll}7 \mathrm{D}-1 & 0.895 & 0.898 & 0.680 & 0.542 & 1.000 & 0.943\end{array}$

$\begin{array}{llllllll}7 \mathrm{D}-2 & 0.865 & 0.872 & 0.713 & 0.579 & 0.943 & 1.000\end{array}$

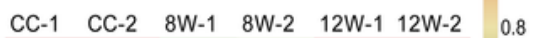

$\begin{array}{llllllll}\text { CC-1 } & 1.000 & 0.994 & 0.579 & 0.670 & 0.970 & 0.964\end{array}$

\begin{tabular}{l|lll|l|l|l|l} 
CC-2 & 0.994 & 1.000 & 0.629 & 0.726 & 0.979 & 0.974
\end{tabular}

$\begin{array}{llllllll}8 \mathrm{~W}-1 & 0.579 & 0.629 & 1.000 & 0.943 & 0.658 & 0.606\end{array}$

$\begin{array}{llllllll}8 \mathrm{~W}-2 & 0.670 & 0.726 & 0.943 & 1.000 & 0.755 & 0.729\end{array}$

$\begin{array}{lllll}12 \mathrm{~W}-1 & 0.970 & 0.979 & 0.658 & 0.755\end{array}$

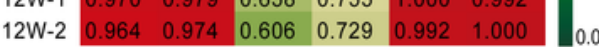

\section{C}
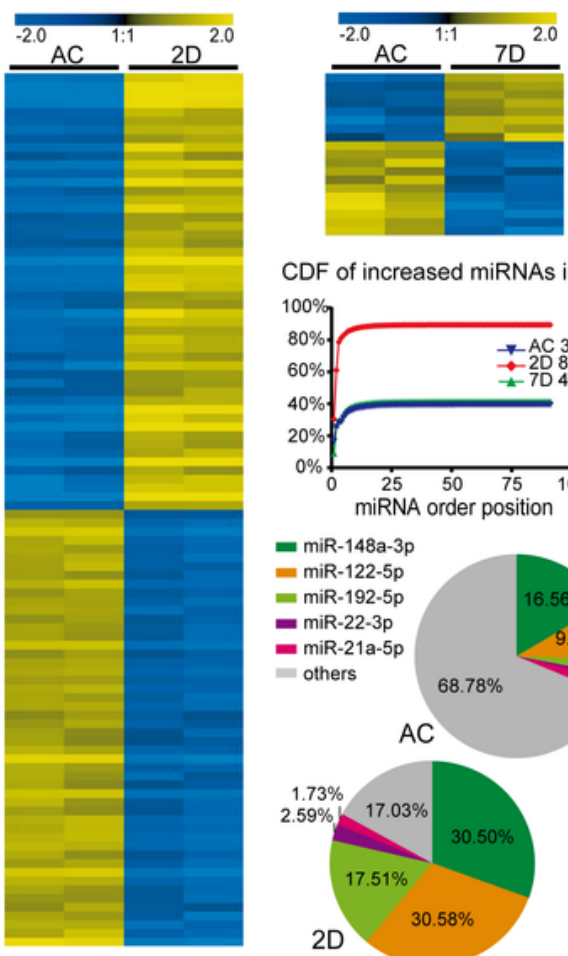

CDF of increased miRNAs in ALI
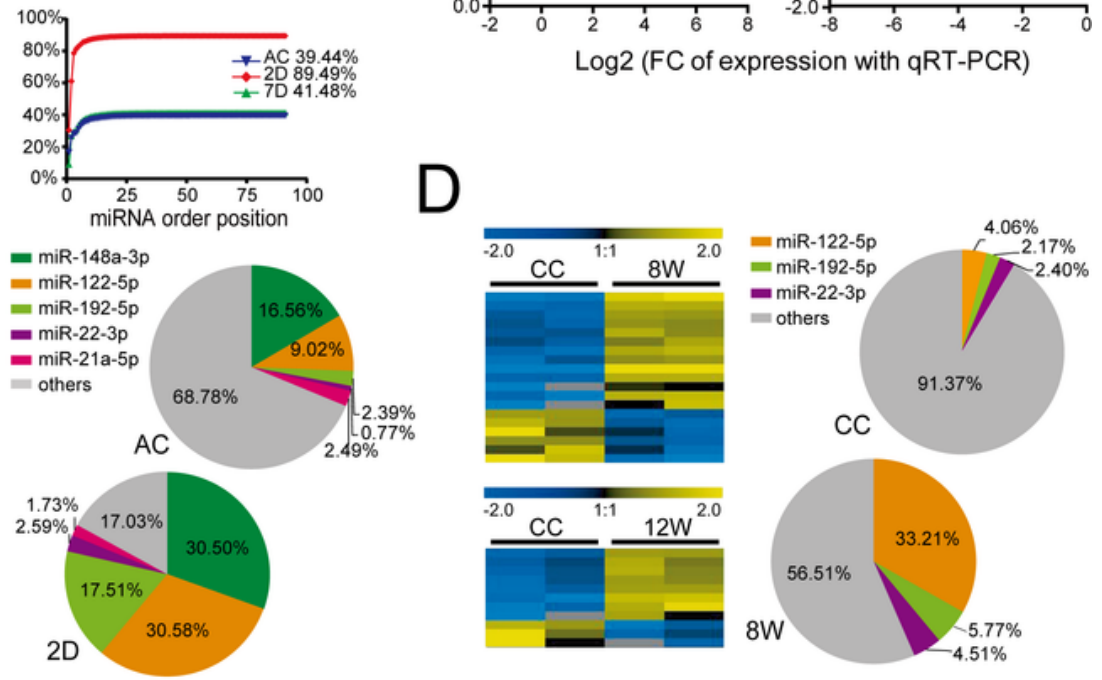

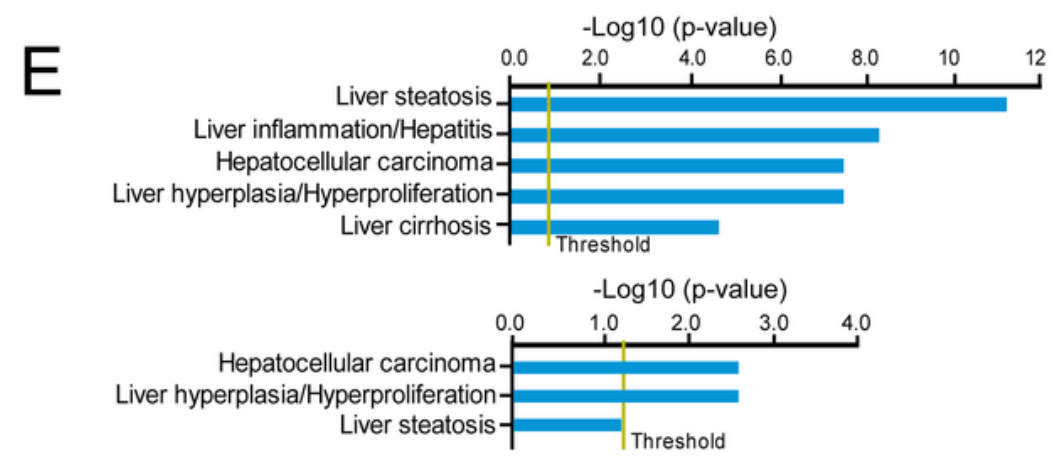

Figure 4

Differentially expressed miRNA in serum sEVs from ALI and CLI mice and the biological significance. (A) Pearson correlation coefficient between samples from ALI and CLI. Correlation coefficient values were labeled in the heat map; red or green color represents high or low correlation, respectively. (B) Validation 
of RNA-seq data by qRT-PCR for miRNAs from ALI and CLI. (C) The heatmap for the differentially expressed miRNAs in the ALI injury stage (2D vs. AC) and recovery stage (7D vs. AC); the cumulative distribution frequency (CDF) plot for the increased miRNAs in ALI; and pie charts illustrated the proportion of the top five up-regulated miRNAs in AC and 2D groups. (D) The heatmap for the differentially expressed miRNAs in the CLI injury stage ( $8 \mathrm{~W}$ vs. CC) and recovery stage ( $12 \mathrm{~W}$ vs. CC). The pie charts illustrated the proportion of the top three up-regulated miRNAs in in CC and $8 \mathrm{~W}$ groups. (E) The top hepatotoxicities for the differentially expressed serum sEV miRNAs in ALI (top), and the differentially expressed serum sEV miRNAs in CLI (bottom) as cataloged by IPA-Tox Functions analysis. D, day; W, week; AC, control for ALI; CC, control for CLI. 


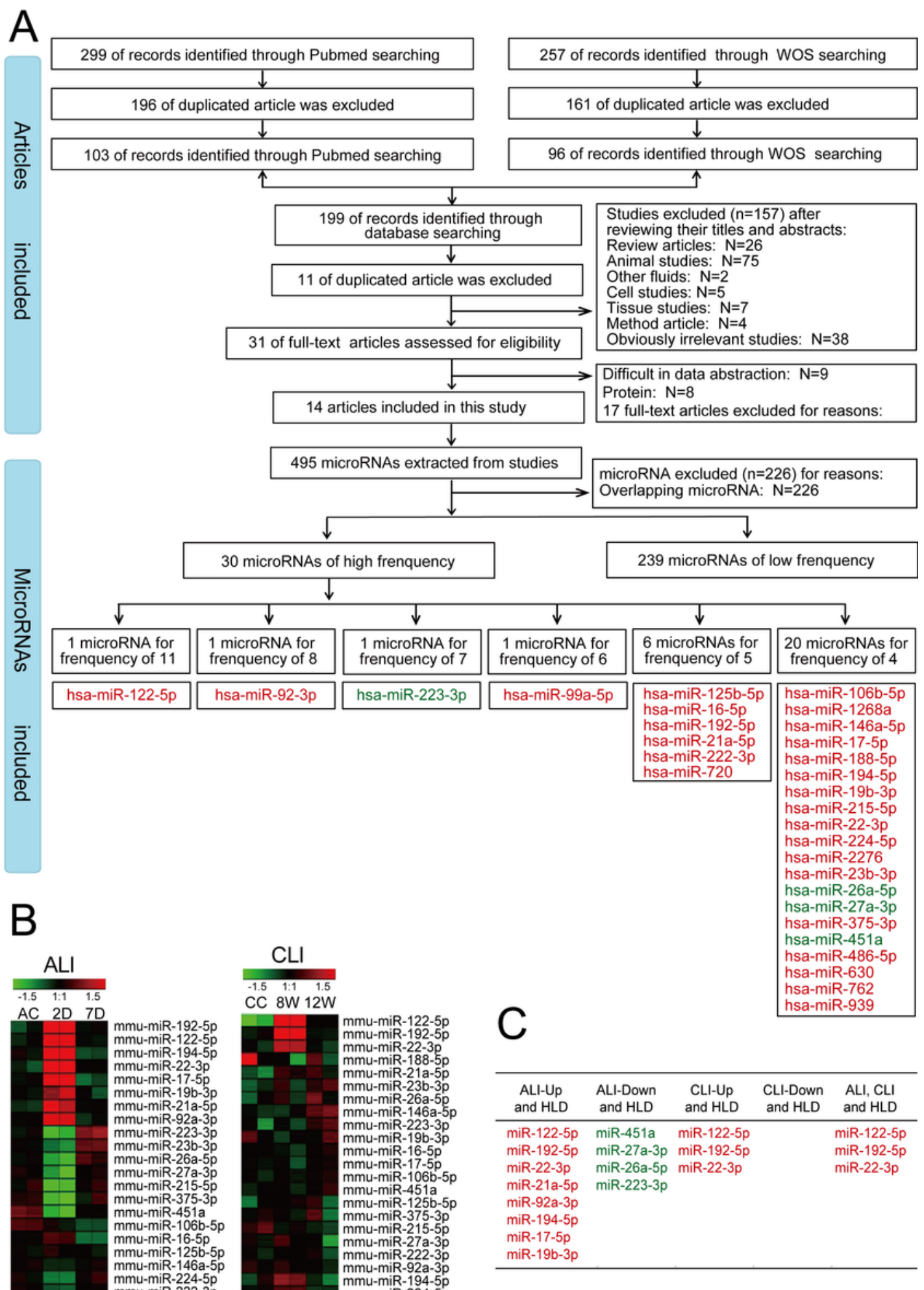

\section{Figure 5}

Differentially expressed circulating miRNAs in human liver disease (HLD) and the common miRNAs in mice. (A) Flow chart of study selection and miRNA screening. (B) Heatmap for the expression of highfrequency HLD circulating miRNAs in ALI and CLI serum sEVs. (C) Common miRNAs in the serum sEVs from mice ALI and CLI, and in the circulation of HLD. The red font, up-regulated miRNAs; the green font, down-regulated miRNAs. ALI-Up, ALI-induced up-regulated miRNAs; ALI-Down, ALI-induced down- 
regulated miRNAs; CLI-Up, CLI-induced up-regulated miRNAs; CLI-Down, CLI-induced down-regulated miRNAs; AC, control for ALI; CC, control for CLI.
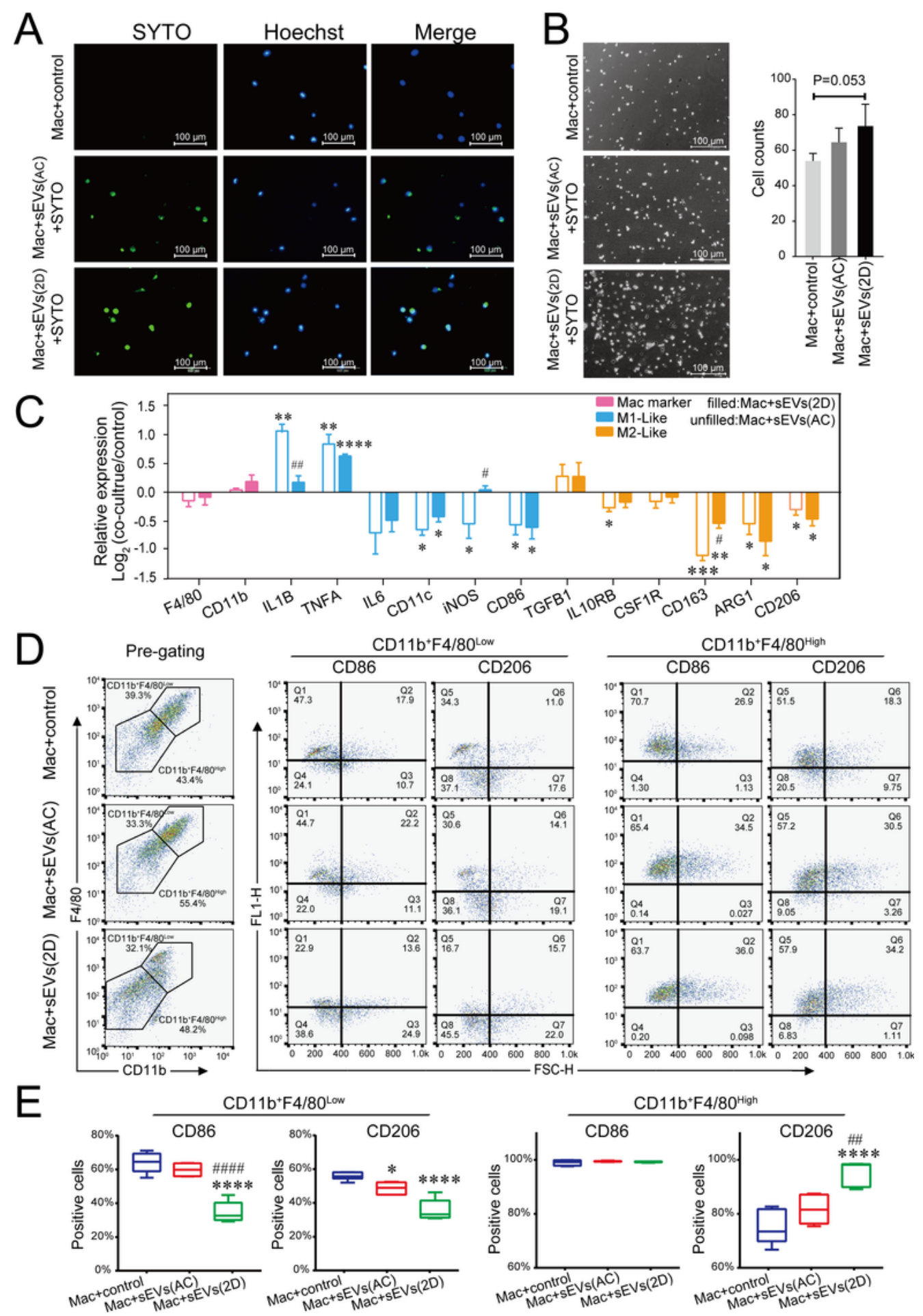

Figure 6

Uptake of serum sEVs by hepatic macrophage and subsequent reprogramming. (A) The uptake of SYTOlabeled serum sEVs from normal (AC) or ALI (2D) mice by primary hepatic macrophages. (B) Hepatic macrophages incubated with AC or 2D serum sEVs for $24 \mathrm{~h}$, and the number of attached cells per $200 \times$ 
field. (C) Expression of M1- and M2-like cell surface markers, and cytokines in hepatic macrophages incubated with mice serum sEVs. The unfilled column represents macrophages incubated with AC sEVs, and the filled column represents macrophages incubated with 2D sEVs. Compared with untreated control, ${ }^{*} \mathrm{p}<0.05,{ }^{* \star} \mathrm{p}<0.01,{ }^{* \star *} \mathrm{p}<0.001,{ }^{* \star \star *} \mathrm{p}<0.0001$; compared with AC sEVs treatment, \#p $<0.05, \# \# p<$ 0.01. (D) Macrophages were defined as CD11b+F4/80Low and CD11b+F4/80High subgroups.

Reresentative images showed the percentage of CD86 and CD206 positive cells in each subgroup with the treatment of control, AC sEVs, and 2D sEVs. (E) CD86 and CD206 positive cells in each subgroup.

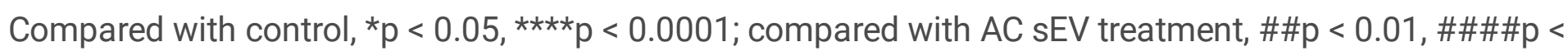
0.0001. Mac, macrophage; Scale bar $=100 \mu \mathrm{m}$. D, day; W, week; AC, control for ALl; CC, control for CLI.

\section{Supplementary Files}

This is a list of supplementary files associated with this preprint. Click to download.

- Supportinglnformation.doc

- SupportingTable1.xls

- SupportingTable2.xls

- SupportingTable3.xls

- SupportingTable4.xls

- SupportingTable5.xls

- SupportingTable6.xls

- SupportingTable7.xls

- SupportingTable8.doc 